PSFC/JA-08-15

\title{
Magnetic topology effects on Alcator C-Mod scrape- off layer flow
}

\author{
Simakov, A.N.*, Catto, P.J, LaBombard, B. and Glasser, A.H.*
}

May 2008

*Los Alamos National Laboratory, Los Alamos

\section{Plasma Science and Fusion Center Massachusetts Institute of Technology Cambridge MA 02139 USA}

This work was supported by the U.S. Department of Energy, Grant No. DE-FG02-91ER54109. Reproduction, translation, publication, use and disposal, in whole or in part, by or for the United States government is permitted.

Submitted for publication in Plasma Physics and Control. Fusion (May 2008) 


\title{
Magnetic topology effects on Alcator C-Mod scrape-off layer flow
}

\author{
Andrei N. Simakov \\ Los Alamos National Laboratory, Los Alamos, New Mexico 87545, USA \\ Peter J. Catto and B. LaBombard \\ MIT Plasma Science and Fusion Center, Cambridge, Massachusetts 02139, USA \\ Alan H. Glasser \\ Los Alamos National Laboratory, Los Alamos, New Mexico 87545, USA
}

(May 1, 2008)

\begin{abstract}
Recent interest in the experimental study of tokamak plasma flow for different magnetic field geometries calls for theoretical understanding of the effects of tokamak magnetic topology changes on the flow. The consequences of total magnetic field reversal and/or X-point reversal on divergence-free plasma flow within magnetic flux surfaces is considered and the results are applied to interpret recent Alcator C-Mod scrape-off layer (SOL) flow measurements.
\end{abstract}




\section{Introduction}

Plasma flow in a tokamak can be beneficial in many ways and is therefore important to understand. Strong plasma rotation can stabilize resistive wall modes ${ }^{1}$; and sheared plasma flow can enhance global plasma stability and increase the achievable ratio of plasma kinetic pressure to magnetic pressure by influencing mode coupling. In particular, sheared flow can increase the threshold for triggering neoclassical tearing modes. ${ }^{2}$ Sheared flow is also known to enhance particle and heat confinement by suppressing and regulating turbulent transport. ${ }^{3}$ In addition, plasma flow in a tokamak scrape-off layer (SOL) is believed to affect SOL transport of neutrals and impurities $^{4}$ as well as the inventory of tritium and products of fusion reactions, influence material migration, ${ }^{5}$ and set boundary conditions for the tokamak core plasma rotation. ${ }^{6}$

In the last few years measurements of the component of the SOL flow parallel to the magnetic field have been made in a number of tokamaks throughout the world, including Alcator C-Mod, ${ }^{6}$ JT-60U, ${ }^{7}$ JET,${ }^{8}$ TCV, ${ }^{9}$ and Tore Supra. ${ }^{10}$ The parallel flow was measured using Mach probes situated at different poloidal locations, so that poloidal variation of the flow could be studied, for different magnetic field topologies. In particular, for diverted tokamaks, measurements were made for lower single null (LSN), upper single null (USN), and double null (DN) operation. In addition, measurements were made for both possible directions of toroidal magnetic field and plasma current (which are usually kept co-directed to preserve magnetic field helicity).

The measured flow is found to be strong, especially at the inner (high field side) far SOL where it may possibly reach Mach numbers of order unity. Moreover, the SOL flow can have strong poloidal variation. For diverted discharges, the inner SOL flow 
has a tendency to remain directed towards an inner divertor, reversing direction for X-point reversal, but independent of the total magnetic field direction. Meanwhile, the outer (low field side) SOL flow tends to be co-current and to reverse for total magnetic field reversal, independent of the X-point position.

The basic understanding that emerges from these measurements is that the SOL flow has two components: an "up-down symmetric" component, which is insensitive to the LSN/USN/DN topology, but cares about the direction of the total magnetic field; and an "up-down asymmetric" component, which responds primarily to the LSN/USN/DN magnetic field topology. The symmetric component is normally assumed to be associated with the nearly divergence-free nature of plasma flow within a magnetic flux surface, which requires parallel ion flow to close ion diamagnetic and $\boldsymbol{E} \times \boldsymbol{B}$ flows. The asymmetric component is assumed to be connected with the strongly poloidally asymmetric "ballooning" nature of radial plasma transport, which results in a local increase in plasma pressure on open field lines about the outer midplane with a corresponding parallel plasma flow directed from the outer midplane toward both inner and outer divertors. Although certain aspects of this basic understanding have been confirmed by numerical modeling results, see e.g., ${ }^{11,12}$ some detailed questions about the SOL flow remain unanswered. In particular, ${ }^{11}$ did not investigate the effects of magnetic topology changes and neglected particle drift effects; and ${ }^{12}$ did not consider X-point reversal and neglected heat flux contributions to the gyroviscosity as well as the perpendicular viscosity, thereby obtaining a questionable radial electric field.

The work presented here uses a simple model ${ }^{13}$ of divergence-free plasma flow within a magnetic flux surface, having symmetric and asymmetric components. It predicts plasma flow changes resulting from X-point reversal and/or total magnetic 
field reversal (Sec. II) and interprets recent Alcator C-Mod SOL flow measurements (Sec. III). Our findings are summarized and conclusions are presented in Sec. IV.

\section{Flow symmetries for X-point and/or $B$ field reversal}

Divergence-free plasma flow in an axisymmetric tokamak is generally an excellent approximation within the magnetic flux surfaces that reside inside the separatrix. On the open field lines outside the separatrix, however, plasma flow can exhibit a strong divergence, particularly in regions where ionization and/or cross-field transport is balanced by parallel flows to the divertor surfaces. In addition, in many tokamaks, a phenomenon of main-chamber recycling takes place in which radial plasma fluxes onto wall surfaces are balanced by recycling and associated ionization sources. Yet in this case, large-scale flow within the flux surfaces is not significantly affected since material flux that is convected radially to the wall must be balanced by local ionization sources.

In view of this picture, we expect the strong, large-scale plasma flows that are observed in the tokamak edge, which are far removed from divertor surfaces and which connect from outer to inner midplanes in single-null discharges, to be well approximated by a divergence-free flow field,

$$
\boldsymbol{V}=\omega R^{2} \boldsymbol{\nabla} \zeta+\frac{K(\psi)}{n} \boldsymbol{B},
$$

where $\omega$ is a toroidal rotation frequency, $R$ is the cylindrical radial coordinate, $\zeta$ is the toroidal angle variable, $K(\psi)$ is a function of the poloidal magnetic flux coordinate $\psi, n$ is plasma density, and the tokamak magnetic field is taken in the form $\boldsymbol{B}=I(\psi) \boldsymbol{\nabla} \zeta+\boldsymbol{\nabla} \zeta \times \boldsymbol{\nabla} \psi$. In double-null configurations, SOL flux tubes do not connect outer and inner midplanes. Nevertheless, expression (1) is valid for such configurations inside the separatrix and by continuity we expect it to approximately 
hold just outside the separatrix as well. Based on these arguments, we will assume that (1) approximately holds for near-SOL flows in both single and double-null cases and use it to study whether we can simply explain the observed tokamak flow symmetries related to X-point and/or the total magnetic field reversal. It will be apparent shortly that our simple model based on (1) describes Alcator C-Mod flow-symmetry observations very well, thereby justifying a posteriori our use of (1) to explore the phenomenon.

We allow the flow in (1) to have both symmetric (s) (i.e. independent of the X-point position) and asymmetric (a) (i.e. dependent on the X-point position) contributions and briefly summarize the relevant tokamak flow symmetries, namely those related to X-point and/or the total magnetic field reversal, as obtained in. ${ }^{13}$ By definition, the flow in an up-down symmetric DN configuration has only a symmetric contribution that we write as

$$
\boldsymbol{V}_{D}=\omega_{s} R^{2} \boldsymbol{\nabla} \zeta+\frac{K_{s}(\psi)}{n} \boldsymbol{B}
$$

The flow in the LSN and USN configurations has both symmetric and asymmetric contributions and can be written for cross-sections of the same shape as

$$
\boldsymbol{V}_{L}=\left(\omega_{s}+\omega_{a}\right) R^{2} \boldsymbol{\nabla} \zeta+\frac{K_{s}(\psi)+K_{a}(\psi)}{n} \boldsymbol{B}
$$

and

$$
\boldsymbol{V}_{U}=\left(\omega_{s}-\omega_{a}\right) R^{2} \nabla \zeta+\frac{K_{s}(\psi)-K_{a}(\psi)}{n} \boldsymbol{B}
$$

respectively. Clearly, X-point reversal reverses the sign of the asymmetric flow portion, leaving the symmetric portion unchanged.

Reversing (R) the total magnetic field, $\boldsymbol{B}_{R}=-\boldsymbol{B}$, in a DN configuration is equivalent to simply turning the up-down symmetric tokamak over, thereby reversing 
the entire flow to obtain

$$
\boldsymbol{V}_{D}^{R}=-\omega_{s} R^{2} \nabla \zeta+\frac{K_{s}(\psi)}{n} \boldsymbol{B}_{R}=-\omega_{s} R^{2} \nabla \zeta-\frac{K_{s}(\psi)}{n} \boldsymbol{B}
$$

However, turning an up-down asymmetric tokamak over reverses not only the total magnetic field but also the X-point. Therefore, to only reverse the total magnetic field in an up-down asymmetric tokamak we have to both reverse the X-point (reversing the asymmetric flow portion) and turn the tokamak over (reversing the total flow), thereby reversing the symmetric flow portion to find

$$
\begin{aligned}
& \boldsymbol{V}_{L}^{R}=-\left(\omega_{s}-\omega_{a}\right) R^{2} \nabla \zeta-\frac{K_{s}(\psi)-K_{a}(\psi)}{n} \boldsymbol{B}, \\
& \boldsymbol{V}_{U}^{R}=-\left(\omega_{s}+\omega_{a}\right) R^{2} \boldsymbol{\nabla} \zeta-\frac{K_{s}(\psi)+K_{a}(\psi)}{n} \boldsymbol{B} .
\end{aligned}
$$

Flow symmetry properties (2) - (6) associated with X-point and total $\boldsymbol{B}$ reversals are clearly independent of the exact expressions for $\omega_{s}, \omega_{a}, K_{s}$, and $K_{a}$. The flux functions $K_{s}$ and $K_{a}$ can be obtained from neoclassical theory on closed flux surfaces (i.e. inside the separatrix) for different collisionality regimes, ${ }^{14,15}$ but are otherwise unavailable. Expressions for $\omega_{s}$ and $\omega_{a}$ can be easily obtained for all collisionality regimes from the ion equation of motion for both closed and open flux surfaces without invoking neoclassical theory assumptions (except for axisymmetry) to find

$$
\omega_{s}=-c\left(\left.\frac{\partial \varphi}{\partial \psi}\right|_{s}+\frac{1}{e n} \frac{\partial p_{i}}{\partial \psi}\right), \quad \omega_{a}=-\left.c \frac{\partial \varphi}{\partial \psi}\right|_{a},
$$

where $c$ is the speed of light, $e$ is the magnitude of electron charge, $\varphi$ is electrostatic potential, and $p_{i}$ is the ion pressure. In (7) $\omega_{a}$ does not contain the pressure gradient term since for matched discharges the density and ion temperature profiles are lowest order flux functions and therefore, by definition have to be the same in LSN and USN and for reversed $\boldsymbol{B}$ operation. Consequently, only the electric field term in $\omega$ can have an asymmetric contribution. 


\section{Analysis of Alcator C-Mod SOL flow measurements}

\section{A. Flow symmetries}

Recent Alcator C-Mod SOL parallel flow measurements were performed for LSN and USN discharges with "normal" and "reversed" $\boldsymbol{B}$ (ion grad- $B$ drift towards the lower and the upper divertors, respectively) for closely matched plasma poloidal crosssections with the same line-average density, as well as other discharge parameters. A few $D N$ flow results for "normal" and "reversed" $\boldsymbol{B}$ are also available. Flow measurements were performed with scanning Mach probes situated $5.8 \mathrm{~cm}$ below the plasma midplane on the high field side and $11 \mathrm{~cm}$ above the plasma midplane on the low field side, so that parallel flow at these two different positions is available for a number of SOL flux surfaces. Typical error bars, based on the shot-to-shot variation in the parallel flow measurements, are in the range of $\pm 10 \mathrm{~km} / \mathrm{s}$ for the high-field side SOL flow measurements and $\pm 5 \mathrm{~km} / \mathrm{s}$ for the low-field side SOL flow measurements. Densities and electron temperatures are also deduced from the probe measurements. The diagnostics, probe design, as well as the data analysis procedure are described in detail in. ${ }^{6}$ Due to inaccuracies in the probe geometry, density measurements on the high-field side may be systematically high or low by $\pm 50 \%$. Otherwise, typical density and temperature measurements error bars are in the range of $\pm 10 \%$.

To determine the four unknowns $\omega_{s}, \omega_{a}, K_{s}$, and $K_{a}$ from (3), (4), and (6) only measurements for two magnetic topologies need be employed, assuming $\omega_{s}$ and $\omega_{a}$ are approximate flux functions. The measured density (and electron temperature) profiles support the lowest order flux function approximation. It can be seen from

Fig. 1 that for LSN and USN cases electron density varies by at most a factor of two or less between locations of the low and high field side probes (i.e. over a length 
$\sim q R \sim 3.4 \mathrm{~m})$. At the same time, it varies by a factor of two in the radial direction over a distance of only about $4 \mathrm{~mm}$, so the radial variation is about 850 times faster than the parallel variation. Therefore, for many practical purposes (including our simple lowest order theory) the flux function approximation should be excellent.

Consequently, the symmetric and asymmetric portions of $\omega$ and $K$ can be evaluated using (3), (4), and (6) in four different ways from the following measurements: (i) $V_{\| L}$ and $V_{\| U}$, (ii) $V_{\| L}^{R}$ and $V_{\| U}^{R}$, (iii) $V_{\| L}$ and $V_{\| L}^{R}$, and (iv) $V_{\| U}$ and $V_{\| U}^{R}$. As a qualitative check, $\omega_{s}$ and $K_{s}$ can be also evaluated using (2) and (5) from $V_{\| D}$ and $V_{\| D}^{R}$ (this check would be quantitative if the flux surfaces of DN discharges were better matched to be the ones recovered when field asymmetry is removed). Close matching of the radial profiles of $\omega_{s}, \omega_{a}, K_{s}$, and $K_{a}$ obtained in the four different ways would confirm the correctness of our understanding of the flow response to X-point and/or $\boldsymbol{B}$ reversals in the absence of any complications.

Before beginning our analysis of the flow data we check the symmetry properties of the Alcator C-Mod flow measurements. If the vacuum chamber and the fuelling were up-down symmetric, discharge parameters were ideally matched (including radial profiles), and measurements were in the midplane, then the radial profiles of $V_{\| L}$ and $V_{\| U}^{R}$, and of $V_{\| U}$ and $V_{\| L}^{R}$, would be identical since these two cases would then correspond to turning over Alcator C-Mod. Figure 2 shows the comparison of these quantities on the low and high field sides. The quantity $\rho$ measures the distance from the separatrix into the SOL projected along flux surfaces onto the outer midplane. Except for the high field side $V_{\| U}$ and $V_{\| L}^{R}$ data, the measured parallel flows do not possess the desired symmetry, and the high field side data for $V_{\| U}^{R}$ is possibly questionable. The lack of symmetry in the parallel flow may be due to the different geometries of the upper and lower divertors and other vacuum vessel asymmetries in 
Alcator C-Mod, up-down asymmetries in fuelling and in the Mach probe locations, and inadequate matching of the discharges. Consequently, we anticipate less than ideal results.

Using the plasma SOL flow and density data as well as our knowledge of magnetic field geometry (from EFIT equilibrium reconstruction data) for each shot we can employ $(2)$ - (6) to evaluate $\omega_{s}$ and $K_{s}$ five different ways (including the DN shots) and $\omega_{a}$ and $K_{a}$ four different ways. The results are shown in Fig. 3. Normalization constants are chosen as follows: $B_{0}=4.1 \mathrm{~T}, I_{0}=3.5 \mathrm{~T} \cdot \mathrm{m}$, and $n_{0}=10^{13} \mathrm{~cm}^{-3}$. Solid lines represent quantities obtained from $V_{\| L}$ and $V_{\| U}$, dashed-dotted lines are from $V_{\| L}^{R}$ and $V_{\| U}^{R}$, dotted are from $V_{\| L}$ and $V_{\| L}^{R}$, dashed are from $V_{\| U}$ and $V_{\| U}^{R}$, and dashed-dotted-dotted are from $V_{\| D}$.

If our simple picture of the flow symmetries, as given by (2) - (6), were correct and all the parameters for different magnetic field geometry discharges were ideally matched, all the curves for $\omega_{s}, \omega_{a}, K_{s}$, and $K_{a}$ would be identical. Clearly, there is a complication. The main reason is, that even though the line integrated densities are matched for all the discharges, the SOL radial density profiles are quite different (they differ by factors of two to three) and so the discharges are not ideal matches. Figure 1 illustrates this point by comparing high and low field side density profiles for the discharges of Fig. 2. Shown are density profiles for the LSN configuration with "normal" and "reversed" $\boldsymbol{B}, n_{L}$ and $n_{L}^{R}$, respectively; the USN configuration with "normal" and "reversed" $\boldsymbol{B}, n_{U}$ and $n_{U}^{R}$, respectively; and the DN configuration with "normal" $\boldsymbol{B}, n_{D}$.

We expect $K(\psi) \propto\langle n\rangle_{\theta}$ since when multiplied by $\boldsymbol{B}$ it represents ion flux, with $\langle n\rangle_{\theta}$ the flux surface averaged density. Therefore, $K(\psi)$ is sensitive to differing density profiles, whereas $K(\psi) /\langle n\rangle$ is not. Assuming that $n \approx\langle n\rangle_{\theta}$ we can approximately 
replace $K_{s, a}(\psi) / n$ with $u_{s, a}(\psi)=K_{s, a}(\psi) /\langle n\rangle_{\theta}$ in $(2)-(6)$ and thereby avoid most of the difficulty with unmatched density profiles. The experimental justification for us to "adjust" the local density profiles to conform to a flux-surface average one comes from the clear insensitivity of the measured flow profiles to variation in plasma density. Figure 7 from $^{6}$ shows what happens to the SOL flows as the line-averaged density is changed. Within error bars, the high-field side flows are essentially unchanged for a factor of $\sim 2$ variation in line-averaged density. Therefore, we have good reason to believe that if we could have obtained a set of discharges with precisely matched SOL density profiles, the resulting flow profiles would not be any different (within error bars) from those that employed the flux surface averaged density profile rather than the local one.

Also, replacing the questionable data for $V_{\| U}^{R}$ on the high field side with its counterpart $V_{\| L}$ [recall Fig. $\left.2(\mathrm{~b})\right]$ and evaluating $\omega_{s}, \omega_{a}, u_{s}$, and $u_{a}$ we obtain rather remarkably improved agreement among different curves, as shown in Fig. 4. The impressive agreement (excluding the DN curves since they are only for qualitatively matched flux surfaces) indicates that our model of the flow symmetries with respect to $\mathrm{X}$-point and $\boldsymbol{B}$ reversals is quite good. Some of the remaining differences between the curves is presumably caused by up-down asymmetries of the vacuum vessel, fueling and probe location, and/or poloidal density variation.

\section{B. Radial electric field}

Employing the $\omega_{s}(\rho)$ and $\omega_{a}(\rho)$ profiles shown in Fig. 4 and using (7) we should in principle be able to evaluate the symmetric and asymmetric portions of the radial electric field and consequently the full radial electric field for all the different magnetic field topology configurations discussed, provided the ion pressure and density profiles 
are known (the latter is measured by the probe). This turns out to be non-trivial since (i) the measured profiles of the ion temperature, $T_{i}(\rho)$, are unavailable; and (ii) the pressure and density profiles for the discharges with the different magnetic field topologies are not precisely matched. To overcome these difficulties we (i) assume $T_{i}=$ $T_{e}$ (with the electron temperature, $T_{e}$, being measured); and (ii) use expressions $\boldsymbol{V}=$ $\omega(\psi) R^{2} \nabla \zeta+K(\psi) \boldsymbol{B} / n, \boldsymbol{V}^{R}=-\omega(\psi) R^{2} \boldsymbol{\nabla} \zeta-K(\psi) \boldsymbol{B} / n$, and $\omega(\psi)=c(\partial \rho / \partial \psi)\left[E_{r}-\right.$ $\left.(e n)^{-1}\left(\partial p_{i} / \partial \rho\right)\right]$ together with the high and low field side measurements of the parallel flow velocities and $n$ and $T_{e}$ profiles for each magnetic field configuration (i.e. without splitting quantities into symmetric and asymmetric). We first evaluate $\omega$ and then $E_{r} \equiv-(\partial \varphi / \partial \rho)$ individually for each case, where

$$
E_{r}\left[\frac{\mathrm{V}}{\mathrm{cm}}\right]=\frac{10}{n\left[\mathrm{~cm}^{-3}\right]} \frac{\partial\left\{n\left[\mathrm{~cm}^{-3}\right] T_{e}[\mathrm{eV}]\right\}}{\partial \rho[\mathrm{mm}]}+11.6\left(\frac{\omega I_{0}}{B_{0}}\right)\left[\frac{\mathrm{km}}{\mathrm{s}}\right]\left(\frac{\partial \psi}{\partial \rho}\right)[\mathrm{T} \cdot \mathrm{m}]
$$

is not a flux function because of $\partial \psi / \partial \rho$. For the discharges under consideration $\partial \psi / \partial \rho \approx 0.57 \mathrm{~T} \cdot \mathrm{m}$ for the low field side and $0.45 \mathrm{~T} \cdot \mathrm{m}$ for the high field side, respectively.

The results are shown in Fig. 5. In particular, Figs. 5 (a) and (b) present the low field side and high field side $E_{r}$ for the LSN discharge with "normal" $\boldsymbol{B}$ (solid lines) and USN discharge with "reversed" $\boldsymbol{B}$ (dashed lines), respectively; while Figs. 5 (c) and (d) present the low field side and high field side $E_{r}$ for the USN discharge with "normal" $\boldsymbol{B}$ (solid lines) and LSN discharge with "reversed" $\boldsymbol{B}$ (dashed lines), respectively.

While the solid and the dashed lines are relatively well matched, as expected from the symmetry considerations, they are not in agreement with the experimentally deduced values $E_{r} \sim 40 \mathrm{~V} / \mathrm{cm}$ in the far SOL for all the magnetic field topology configurations considered (see Fig. 11 of $^{6}$ ). Although the Mach and $E_{r}$ probe mea- 
surements have their own difficulties (see e.g. discussion in Sec. 3.3 .3 of $^{6}$ ), the large discrepancies between these measurements and our results shown in Fig. 5 suggest other underlying causes. One of these may be that the assumption $T_{i} \approx T_{e}$ is invalid in the SOL (as is the case in the tokamak pedestal region ${ }^{16,17}$ ) and the procedure for evaluating $E_{r}$ described herein can only be reliable and useful when accurate $T_{i}(\rho)$ profiles are available. The sensitivity of $E_{r}$ to $T_{i}$ arises not just because of the normalization of the flow to the sound speed. The more important effects are the tendency of the symmetric and asymmetric contributions in $\omega$ and $u$ to cancel for normal LSN and reversed USN, and the tendency of the total $\omega$ and $K=u\langle n\rangle_{\theta}$ contributions to the flow to cancel for normal USN and reversed LSN. Hence small errors in these derived quantities lead to large errors in the inferred values of $E_{r}$.

\section{Conclusions}

A simple theory for plasma flow modifications due to the X-point reversal and the total magnetic field reversal is discussed and used to interpret Alcator C-Mod SOL flow measurements. The theory employed is rather basic and summarized by (2) (6). According to these expressions, X-point reversal is expected to cause reversal of the "asymmetric" flow portion, while $\boldsymbol{B}$ reversal is expected to result in reversal of the "symmetric" flow portion. We demonstrate that these expressions are all that is required to analyze recent high and low field side Mach probe SOL flow measurements in the Alcator C-Mod tokamak for LSN, USN, and DN magnetic field configurations with "normal" and "reversed" magnetic field (ion grad- $B$ drift towards the lower and the upper divertors, respectively).

Equations (3), (4), and (6) are used to evaluate the symmetric and asymmetric 
portions of $\omega$ and $K$ in four different ways by using the inboard and outboard probe measurements for the following cases: (i) $V_{\| L}$ and $V_{\| U}$, (ii) $V_{\| L}^{R}$ and $V_{\| U}^{R}$, (iii) $V_{\| L}$ and $V_{\| L}^{R}$, and (iv) $V_{\| U}$ and $V_{\| U}^{R}$. Also, $\omega_{s}$ and $K_{s}$ are evaluated from $V_{\| D}$ by using (2). If the theory were rigorously correct and the discharges were ideally matched then all the curves for $\omega_{s, a}$ and $K_{s, a}$ would be the same. However, the raw agreement is only suggestive as can be seen from Fig 3.

To improve the agreement we must account for the order unity differences in the radial density profiles (see Fig. 1), since the data was taken by matching only the line average density. If the density is assumed to be an approximate flux function and $K(\psi) / n$ is replaced with $u(\psi)$ in $(2)-(6)$ then the agreement between the curves improves dramatically (see Fig. 4), indicating that our model of the flow symmetries is rather good. We expect that the agreement could be further improved by matching the radial density and temperature profiles rather than the line-average density in the discharges with different magnetic topologies.

Knowledge of plasma density and ion temperature profiles allows determination of the radial electric field, as shown in Fig. 5 . The results obtained by assuming $T_{i}=T_{e}$ (since the $T_{i}$ profiles are not available while the $T_{e}$ profiles are) are consistent with our symmetry considerations (e.g. $E_{r}$ for the LSN discharge with "normal" $\boldsymbol{B}$ and USN discharge with "reversed" $\boldsymbol{B}$ are very similar), but disagree with the far SOL experimental measurements, which typically find $E_{r} \sim 40 \mathrm{~V} / \mathrm{cm}$. This leads us to believe that the assumption $T_{i}=T_{e}$ is invalid, as is the case in the tokamak pedestal region, and that the knowledge of accurate $T_{i}$ profiles is essential for determining the radial electric field profiles by our procedure. 


\section{ACKNOWLEDGEMENT}

This research was supported by the U.S. Department of Energy grants DE-AC5206NA-25396 at Los Alamos National Laboratory, DE-FG02-91ER-54109 and DEFC02-99ER-54512 at the Plasma Science and Fusion Center of the Massachusetts Institute of Technology. 


\section{References}

${ }^{1}$ Garofalo A M et al 2002 Phys. Rev. Lett. 89235001

${ }^{2}$ Buttery R J et al 2001 28th EPS Conference on Contr. Fusion and Plasma Physics (Funchal, Portugal, 2001) European Conference Abstracts vol 25A ed C Silva, J Pines, and R Nóbrega (European Physical Society) p 1813

${ }^{3}$ Burrell K H et al 1992 Plasma Phys. Control. Fusion 341859 and references therein

${ }^{4}$ Parker R et al 1997 J. Nucl. Mater. 241-243 1

${ }^{5}$ Pitts R A et al 2005 Plasma Phys. Control. Fusion 47 B303

${ }^{6}$ LaBombard B et al 2004 Nucl. Fusion 441047

${ }^{7}$ Asakura N et al 2000 Phys. Rev. Lett. 843093

${ }^{8}$ Erents S K et al 2004 Plasma Phys. Control. Fusion 461757

${ }^{9}$ Pitts R A et al 2007 J. Nucl. Mater. 363-365 505

${ }^{10}$ Gunn J P et al 2006 Int. Conf. on Research and Applications of Plasmas (OpoleTurawa, Poland, 2005) AIP Conference Proceedings vol 812 ed M J Sadowski, M Dudeck, H-J Hartfuss, and E Pawelec (American Institute of Physics) p 27

${ }^{11}$ Pigarov A Yu, Krasheninnikov S I, and LaBombard B 2006 Contrib. Plasma Phys. 46604

${ }^{12}$ Bonnin X et al 2005 J. Nucl. Mater. 337-339 301

${ }^{13}$ Catto P J and Simakov A N 2006 Phys. Plasmas 13052507

Catto P J and Simakov A N 2007 Phys. Plasmas 14029901 
${ }^{14}$ Hirshman S P and Sigmar D J 1981 Nucl. Fusion 211079

${ }^{15}$ Hinton F L and Hazeltine R D 1976 Rev. Mod. Phys. 48239

${ }^{16}$ Maggi C F et al 2007 Nucl. Fusion 47535

${ }^{17}$ Kagan G and Catto P J 2007 Bull. Am. Phys. Soc. 52326 


\section{FIGURE CAPTIONS}

Figure 1. SOL plasma density profiles on the (a), (c) low and (b), (d) high field sides with solid lines for LSN configuration densities $n_{L}$ ("normal" $\boldsymbol{B}$ ) and $n_{L}^{R}$ ("reversed" $\boldsymbol{B}$ ), dashed lines for USN configuration densities $n_{U}$ ("normal" $\boldsymbol{B}$ ) and $n_{U}^{R}$ ("reversed" $\boldsymbol{B}$ ), and dotted lines for DN configuration density $n_{D}$ ("normal" $\boldsymbol{B}$ ). The normalization factor $n_{0}=10^{13} \mathrm{~cm}^{-3}$. Due to inaccuracies in the probe geometry, density measurements on the high-field side may be systematically high or low by $\pm 50 \%$. Otherwise, typical density measurements error bars are in the range of $\pm 10 \%$.

Figure 2. Comparison of Alcator C-Mod data for $V_{\| L}$ (solid lines) and $V_{\| U}^{R}$ (dashed lines) in (a), (b) and $V_{\| U}$ (solid lines) and $V_{\| L}^{R}$ (dashed lines) in (c), (d) on the low [see (a), (c)] and high [see (b), (d)] field sides. Error bars are only presented for one curve of each pair to avoid cluttering the figure. The errors are identical for the other curve.

Figure 3. Profiles of normalized (a) $\omega_{s}$, (b) $\omega_{a}$, (c) $K_{s}$, and (d) $K_{a}$. Solid lines represent quantities obtained from $V_{\| L}$ and $V_{\| U}$, dashed-dotted lines are from $V_{\| L}^{R}$ and $V_{\| U}^{R}$, dotted from $V_{\| L}$ and $V_{\| L}^{R}$, dashed lines are from $V_{\| U}$ and $V_{\| U}^{R}$, and dashed-dotteddotted from $V_{\| D}$.

Figure 4. Profiles of normalized (a) $\omega_{s}$, (b) $\omega_{a}$, (c) $u_{s}$, and (d) $u_{a}$ using the same labeling as in Fig. 3 .

Figure 5. Low field side (a), (c) and high field side (b), (d) radial electric field 
profiles evaluated for the LSN discharge with "normal" $\boldsymbol{B}$ [(a), (b), solid lines], USN discharge with "reversed" $\boldsymbol{B}$ [(a), (b), dashed lines], USN discharge with "normal" $\boldsymbol{B}[(\mathrm{c}),(\mathrm{d})$, solid lines], and LSN discharge with "reversed" $\boldsymbol{B}$ [(c), (d), dashed lines]. 


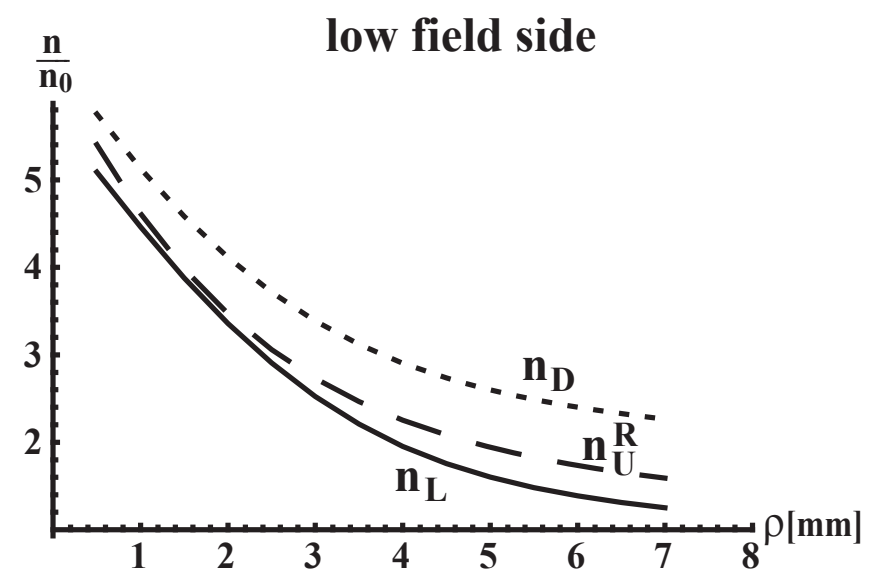

(a)

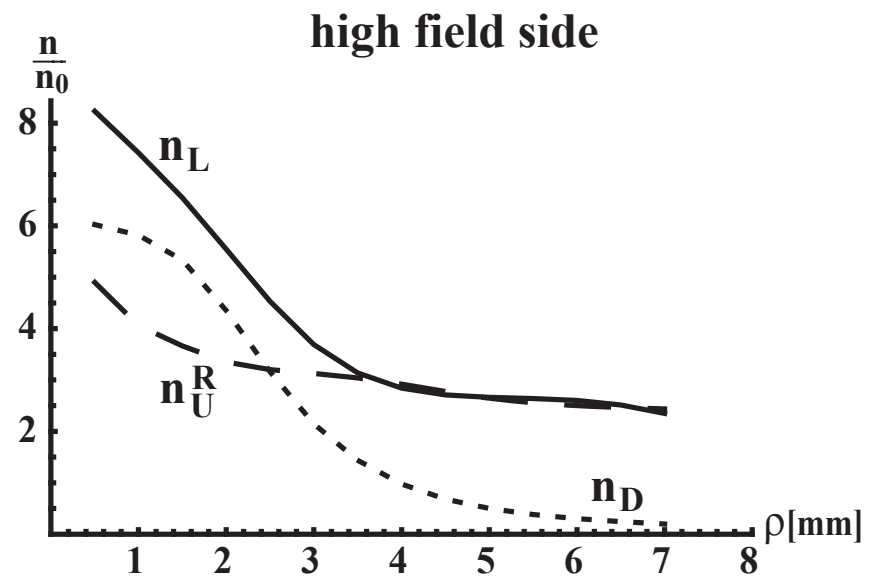

(b)

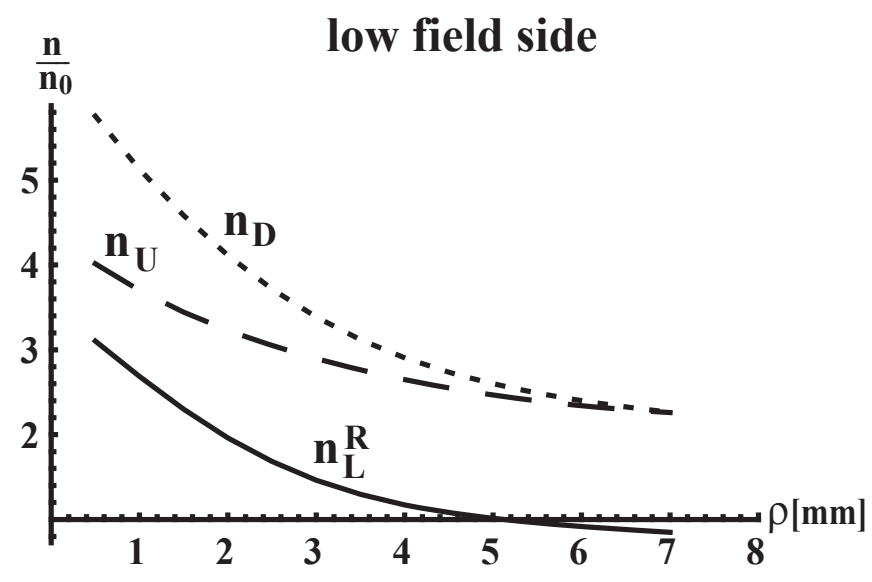

(c)

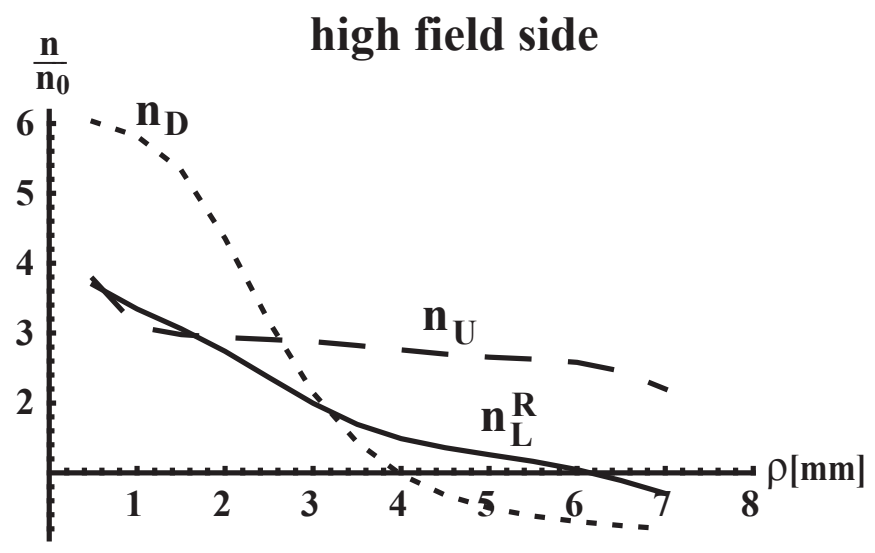

(d)

Figure 1. 


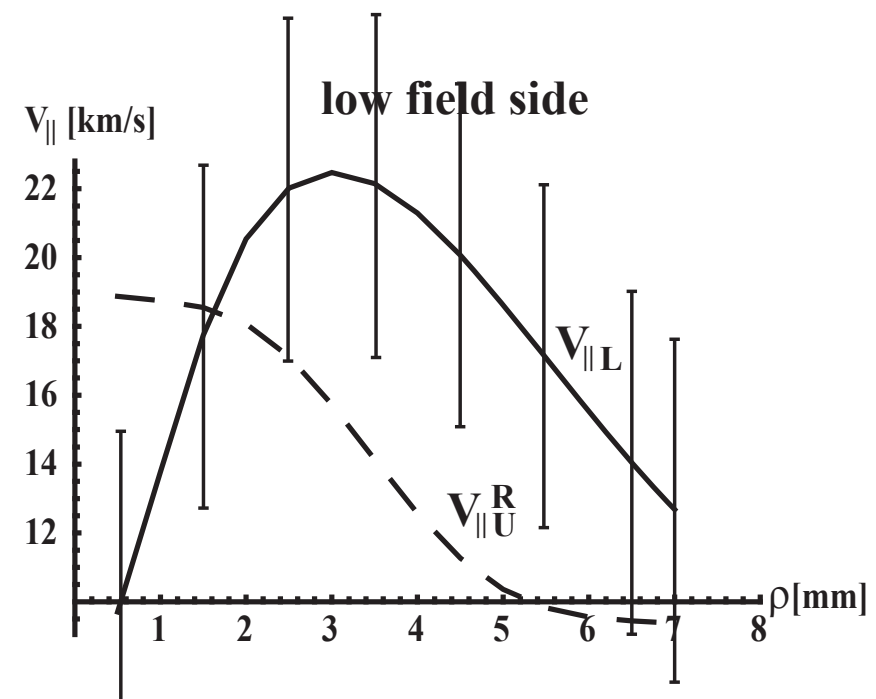

(a)

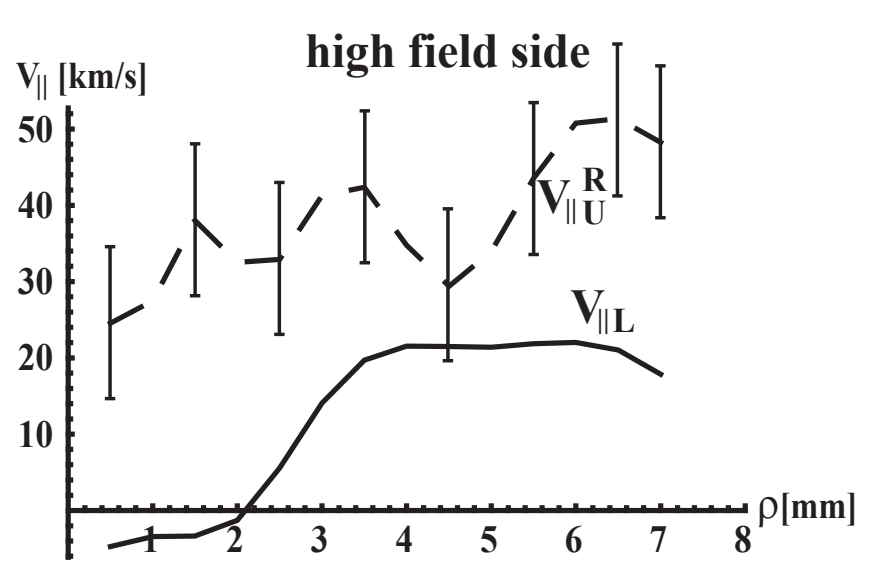

(b)
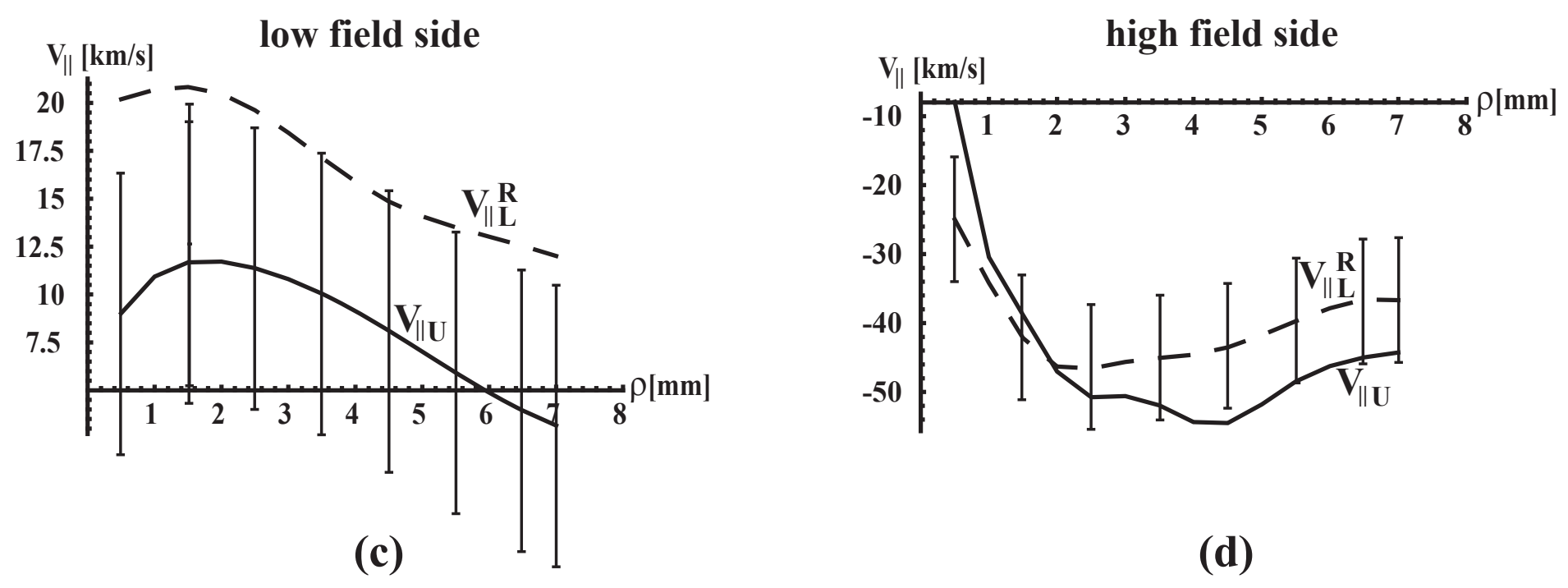

Figure 2. 


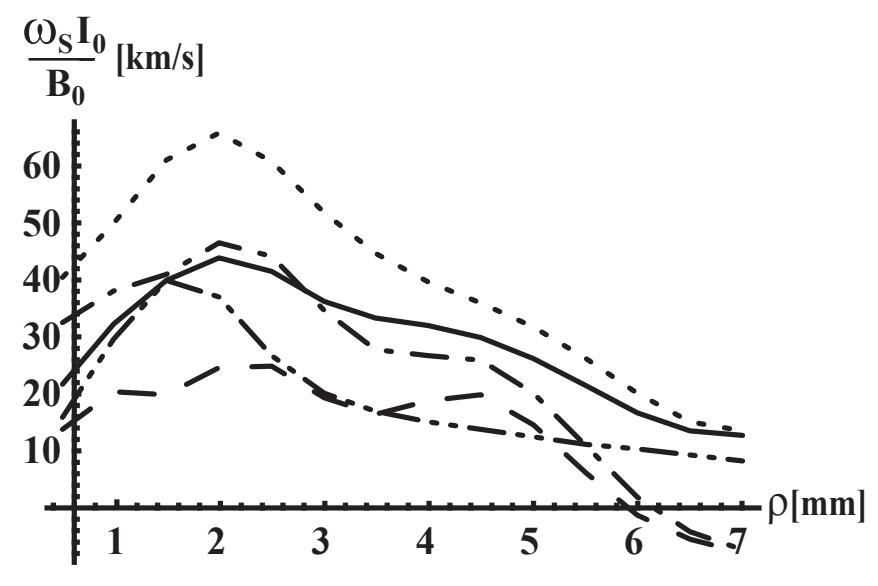

(a)

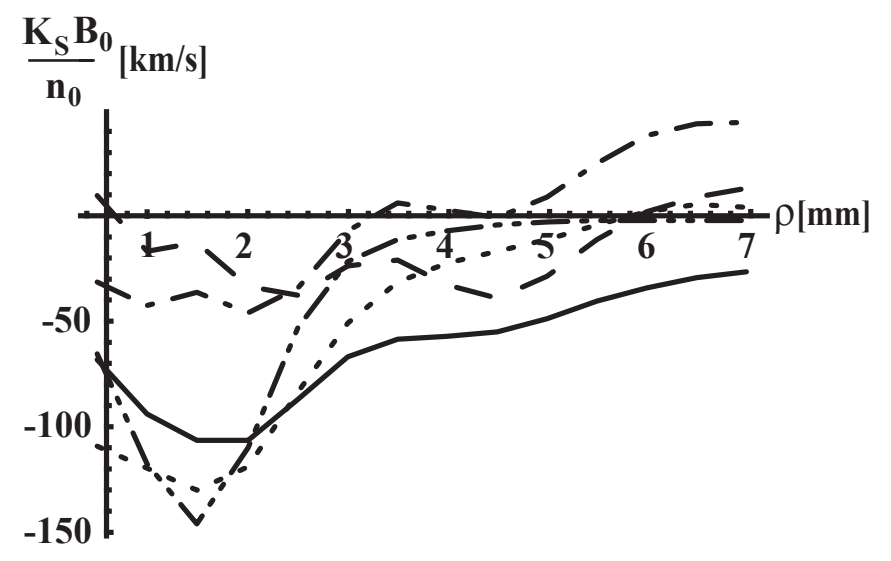

(c)

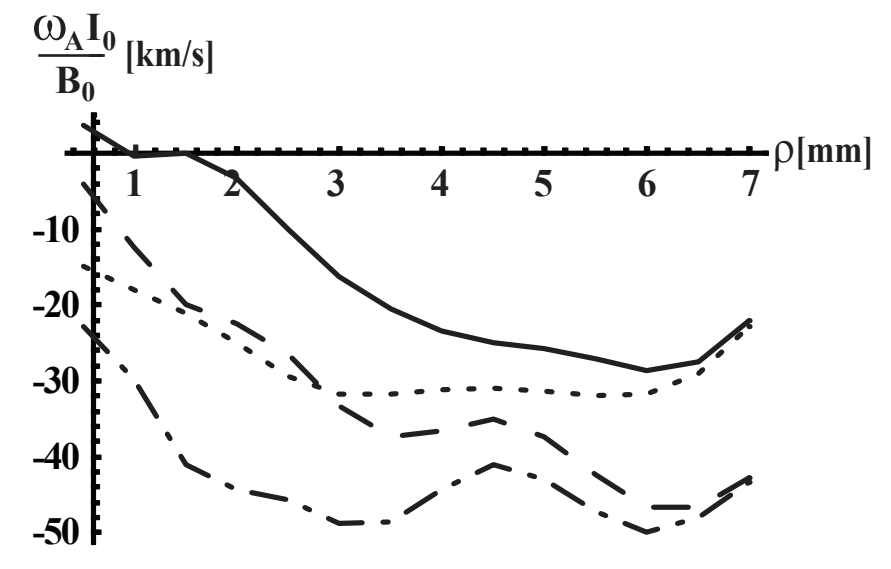

(b)

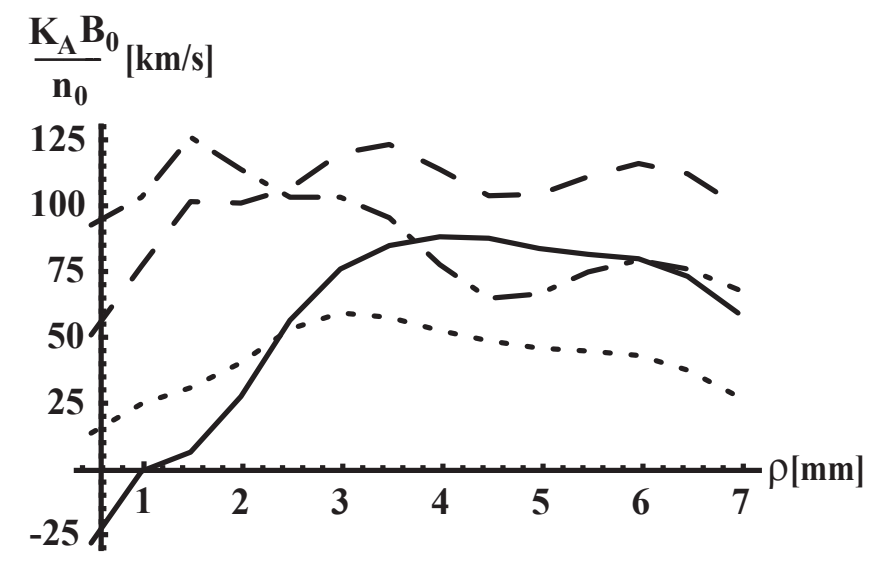

(d)

Figure 3. 


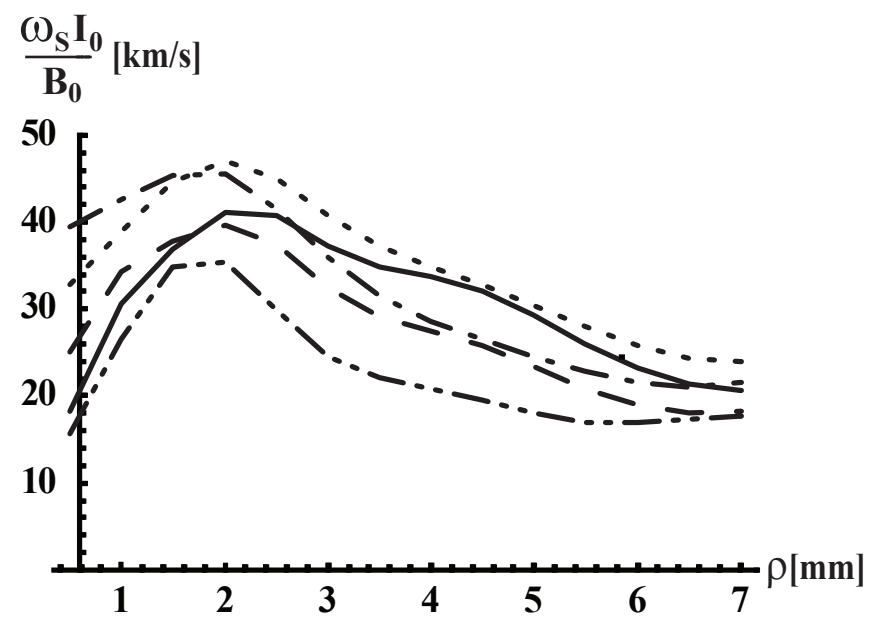

(a)

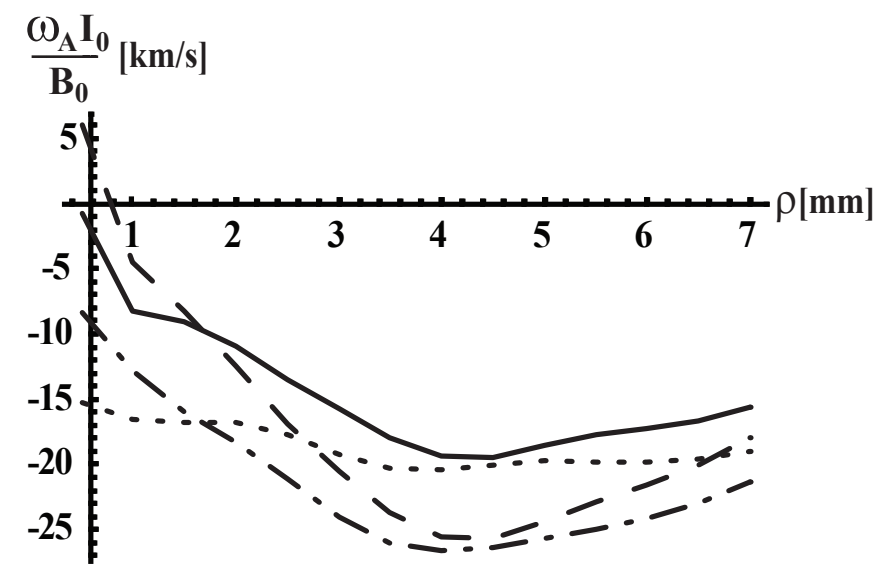

(b)

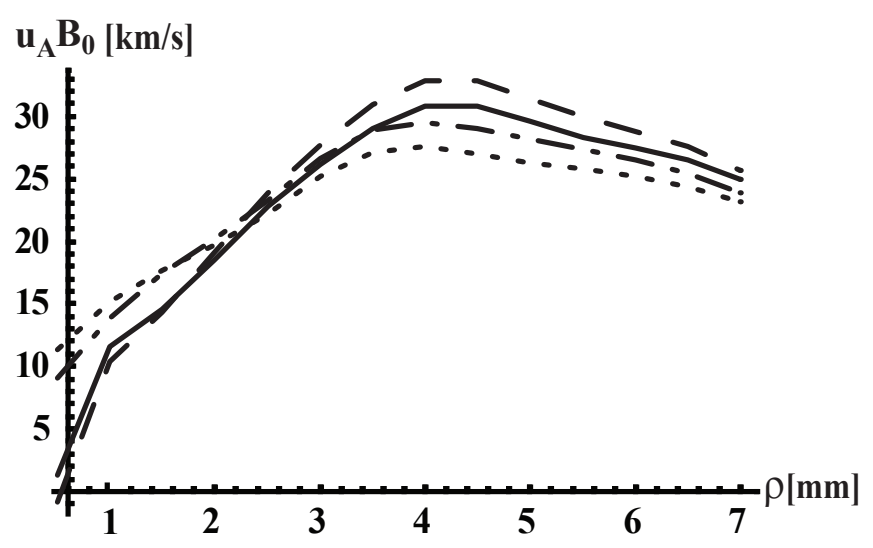

(d)

Figure 4. 


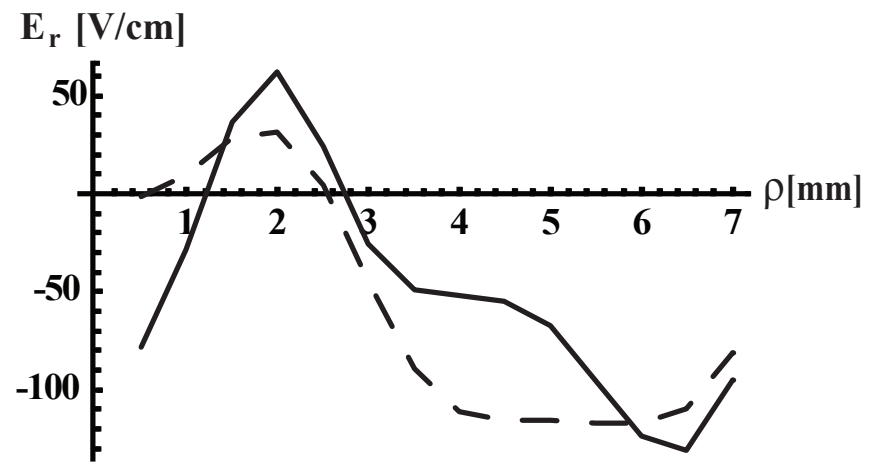

(a)

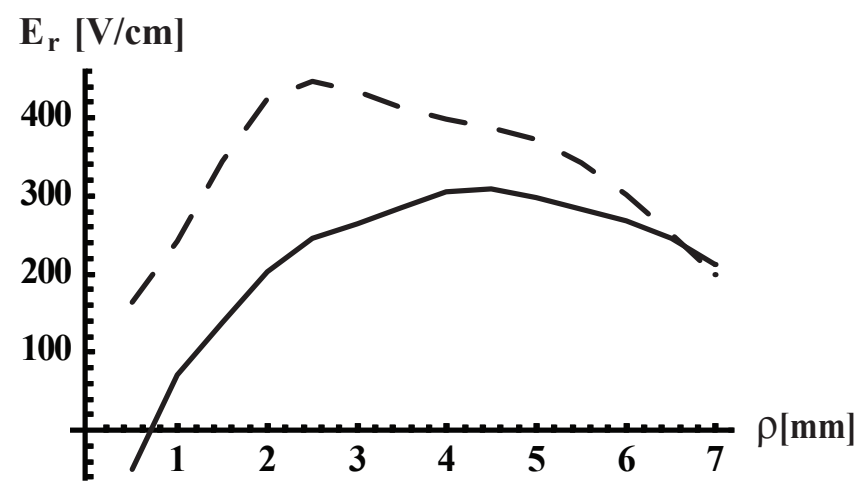

(c)

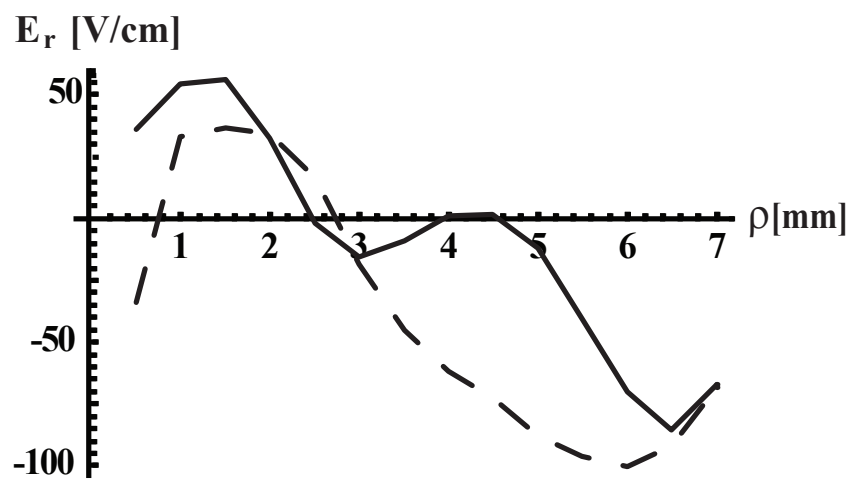

(b)

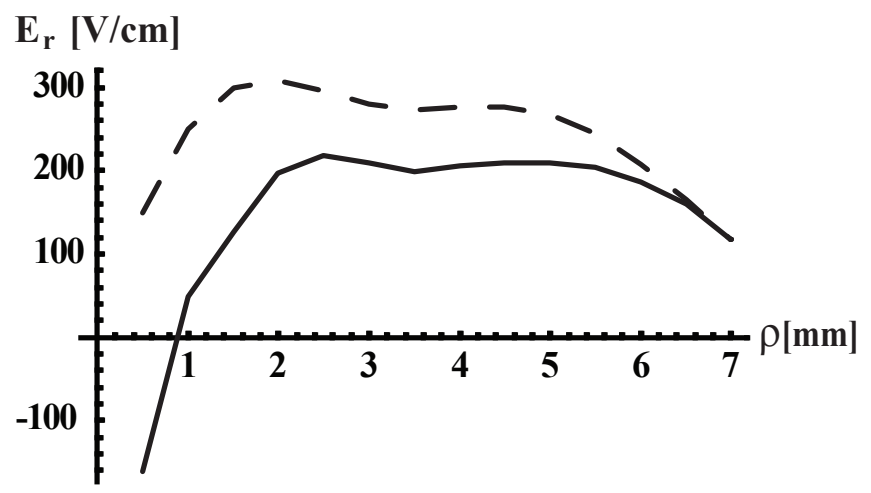

Figure 5. 